\title{
Impact of dissolved humic acid on the bioavailability of acenaphthene and chrysene assessed by membrane-based passive samplers
}

\author{
CHEN Shan, KE RunHui, HUANG ShengBiao, SUN LiWei, ZHA JinMiao \& WANG ZiJian ${ }^{\dagger}$ \\ State Key Laboratory of Environmental Aquatic Chemistry, Research Center for Eco-environmental Science, Chinese Academy of \\ Sciences, Beijing 100085, China
}

Dissolved organic carbon (DOC) is known to reduce the bioavailability of hydrophobic organic compounds (HOCs) in aqueous environments. This reduction occurs as a result of adsorption to DOC, apparently reducing the freely dissolved concentration of HOCs. In the present study, triolein-embedded cellulose acetate membrane (TECAM) and Japanese medaka (Oryzias latipes) were used to measure the uptake of acenaphthene and chrysene in the presence of commercial humic acid (HA) at different concentrations $\left(0-15 \mathrm{mg} \mathrm{C} \cdot \mathrm{L}^{-1}\right)$ under controlled laboratory conditions. Apparent uptake rate constants for PAHs in TECAM and medaka were compared and DOC-water partition coefficients $\left(K_{\mathrm{DOC}} \mathrm{S}\right)$ of two PAHs were calculated with different sampling methods by model fit. Results showed that HA present in water significantly reduced the uptake of PAHs in TECAM and medaka. The obtained values of $\log K_{\mathrm{DOC}}$ of acenaphthene and chrysene measured by TECAM were 4.63 and 5.83 , respectively, whereas biologically determined values were 4.52 and 5.76, respectively. These log $K_{\mathrm{DOC}}$ values were comparable to earlier published $K_{\mathrm{DOC}} s$ toward commercial HA, thereby indicating that TECAM accumulated only the freely dissolved fraction of chemicals and uptake PAHs in a manner similar to that of fish. All these results suggested that the TECAM method can provide a good means for assessing the impact of DOC on bioavailability of PAHs in the aqueous environment.

bioavailability, polycyclic aromatic hydrocarbons, humic acid, biomimetic sampling, partition coefficient, dissolved organic carbon

Dissolved organic carbon (DOC), predominantly humic materials, is ubiquitous in aquatic systems and derives from various sources ${ }^{[1]}$. It has been demonstrated that DOC can adsorb and bind hydrophobic organic compounds (HOCs), thus to a considerable degree, affect their behaviors such as transportation, transformation and distribution in aqueous environments ${ }^{[2]}$. DOC may raise the apparent solubility of HOCs and facilitate their migration in water. On the other hand, the formation of aggregates might be too large or too polar to cross biological membranes ${ }^{[3]}$. Since the bioavailable chemical concentration is often considered to be the freely dissolved chemical concentration in the water as only freely dissolved organic molecules are believed to be able to permeate through the gill membrane $e^{[2,3]}$, and
DOC may significantly decrease the bioavailable concentration then the actual toxicity of HOCs to aquatic organisms. Thus the risk assessment for actual toxicity of HOCs based on the total concentration may be overestimated $^{[2]}$. In order to establish a correct hazard assessment, the impact of DOC on bioavailability of contaminants should be evaluated and to a certain extent quantified. The partition coefficient between DOC and the aqueous phase $\left(K_{\mathrm{DOC}}\right)$ is an important parameter used to describe the distribution of organic pollutants.

\footnotetext{
Received November 21, 2006, accepted April 28, 2007 doi: 10.1007/s11434-007-0378-7

Corresponding author (email: wangzj@rcees.ac.cn)

Supported by the National Natural Science Foundation of China (Grant No. 20507019) and the National Basic Research Program of China (Grant No. 2007CB407304)
} 
The amount of freely dissolved HOC is proportional to $K_{\mathrm{DOC}}$ and the mass of DOC in water, thus the true aqueous concentration of a given HOC can be calculated from obtained $K_{\mathrm{DOC}}$ and total concentration. Methods used to date for measuring the $K_{\mathrm{DOC}}$ in aqueous media include fluorescence quenching, equilibrium dialysis, reverse-phase separation, solid-phase microextraction (SPME), etc. ${ }^{[3,4]}$. Polycyclic aromatic hydrocarbons (PAHs) are a group of persistent contaminants generally known for their global distribution as well as genotoxic, mutagenic, and carcinogenic potentials. They enter water systems via various pathways and have a strong tendency to accumulate in the fat reserves of aquatic organisms due to their strong lipophilicity ${ }^{[5]}$. Therefore, it is of great importance to understand the nature of the interactions between PAHs and organic matter ${ }^{[6,7]}$.

The chemical uptake process of proposed passive sampling method has been recognized as being similar to that of bioconcentration in which HOCs diffuse from the surrounding media across the biological membranes. Therefore, passive sampling devices are rapidly gaining growing acceptance as effective tools to mimic bioconcentration. Since Södergren ${ }^{[8]}$ first proposed the passive sampling technique to accumulate hydrophobic chemicals by dialysis membranes filled with hexane, semipermeable membrane devices have been widely used to monitor trace levels of waterborne organic chemicals $^{[9,10]}$. The most popular one among these devices is the triolein-containing semipermeable membrane device (SPMD) developed by Huckins et al. ${ }^{[11]}$. Moreover, the SPMD-available fraction of contaminants in the media is considered to be similar to the readily bioavailable fraction, therefore this method has been shown to closely predict $K_{\mathrm{DOC}}$ of $\mathrm{PAHs}{ }^{[10]}$.

Previously, we proposed a new type of passive sampling device similar to SPMD, triolein-embedded cellulose acetate membrane (TECAM) as an efficient tool to accumulate organochlorine pesticides (OCPs) and $\mathrm{PAHs}^{[9]}$. TECAM shows some advantages against other sampling methods including low cost, ease of preparation and employment, simple pretreatment procedures, and commonly no additional cleanup step ${ }^{[9,12]}$. However, it is still a hypothesis that only freely dissolved compound could be accessible for extraction by TECAMs. Although the laboratory and field use for uptaking HOCs is successful, TECAM is not used to quantify the effect of DOC on the bioavailability of HOCs in water. Based on these considerations, a negligible depletion extraction method ${ }^{[10]}$ using TECAM (nd-TECAM) as a passive sampler was exploited. The objective of this investigation was to assess to what extent the humic acid (HA) might have affected bioavailability of two PAHs, acenaphthene and chrysene, in aqueous environments. The reductions in the apparent uptake rate constants versus actual uptake rate constant of two PAHs in TECAM were used to calculate their partition coefficients between HA and the aqueous phase (i.e. $K_{\mathrm{DOC}} \mathrm{S}$ ). Then the $K_{\mathrm{DOC}}$ s can be used to estimate bioavailable concentration of two target PAHs in aqueous phase and quantificationally determine the impact of DOC on their bioavailability. Similarly, medaka accumulation experiment under identical condition was performed to validate the use of TECAM as a biomimetic surrogate to predict the bioavailability of HOCs in aquatic systems.

\section{Material and methods}

\subsection{Membrane and experimental organism}

The procedure for preparing TECAM was the same as described previously ${ }^{[14]}$. In TECAM, the triolein drops embedded in the matrix of cellulose acetate polymers and closely adhered with the polymers. Thus, the TECAM consists of two immiscible phases: an outer hydrophilic membrane and inner hydrophobic triolein drops. Small pieces of TECAM $(4 \mathrm{~cm} \times 6 \mathrm{~cm}$, average wet weight $0.159 \mathrm{~g}$ ) were used for all experiments. All TECAMs were washed and kept in Milli-Q water (Millipore, USA) before use. The Japanese medaka (Oryzias latipes) was cultured and maintained for more than five generations in our laboratory before fish were used for experiments. Female medaka selected for bioconcentration experiments was fully mature and outside of spawning period (mean body weight $0.5 \mathrm{~g}$; mean body length, $3 \mathrm{~cm}$ ). They were starved for $24 \mathrm{~h}$ prior to the experiment to clean gut and exclude faeces that may affect the quantity of PAHs bound to HA.

\subsection{Negligible depletion sampling method}

When the extent of extraction measured in the sampling matrix is less than $10 \%$ of the total amount for an analyte, the existing equilibrium between the freely dissolved and the DOC-bound chemicals can be considered as being undisturbed during the measurement, or depletion after extraction is negligible ${ }^{[13]}$. Since experiments in the present study are all carried out in static exposure condition, it is necessary to ensure that the concentra- 
tions of PAHs in exposure solutions remain relatively constant and the existing equilibrium between the freely dissolved and the DOC-bound chemicals is undisturbed during extraction. In the mean time, PAHs are required in the TECAM and medaka to achieve sufficiently high concentration. Therefore, experimental conditions for exposure parameters (i.e. duration and agitation) need to be investigated in detail prior to accumulation experiment.

It is well known that agitation such as shaking can increase the uptake rate constant of TECAM accumulation. In the primary experiment for agitation, 4 conical flasks containing $1 \mathrm{~L}$ of ultrapure water were spiked with 100 ng of two target PAHs (acenaphthene and chrysene) and equilibrated for $24 \mathrm{~h}$. After equilibration, one piece of TECAM was inserted into the solution of each flask and the flask was kept sealed to prevent loss of analytes due to volatilization. The flasks were shaken at speeds of 0 , 50,100 , and $150 \mathrm{r} \cdot \mathrm{min}^{-1}$, respectively at $25^{\circ} \mathrm{C}$. Triplicate samples of TECAMs were taken after $1 \mathrm{~h}$ for every treatment. All the water used in this study was purified by a Milli-Q Gradient system $\left([D O C]<0.1 \mathrm{mg} \cdot \mathrm{L}^{-1}\right)$.

Then the shaking speed was set at $75 \mathrm{r} \cdot \mathrm{min}^{-1}$ accordingly. TECAMs were exposed in the same exposure solutions spiked with the two PAHs to give concentration of individual analyte of $100 \mathrm{ng} \cdot \mathrm{L}^{-1}$ at $25^{\circ} \mathrm{C}$ for 1,2 and $3 \mathrm{~h}$ respectively. Medaka exposure experiment was performed under identical conditions with 2 fish in a flask, kept in steady state. To further examine the loss of target chemicals during the exposure, PAH concentrations in exposure solutions were monitored by liquidliquid extraction method throughout the $1 \mathrm{~h}$ of experimentation.

\subsection{Accumulation kinetics}

Since information of the accumulation kinetics is crucial for sampling, it needs to determine the absorption profiles of PAHs to TECAM and medaka in the exposure experiments. Kinetics experiments of TECAM were conducted by adding one piece of TECAM to 1 liter of test solution spiked with $100 \mathrm{ng} \cdot \mathrm{L}^{-1} \mathrm{PAHs}$ for $1,2,3,6$, and $12 \mathrm{~h}$. All the experiments were performed on a constant temperature-reciprocating shaker with controlled conditions $\left(75 \mathrm{r} \cdot \min ^{-1}, 25^{\circ} \mathrm{C}\right)$. The uptake kinetics experiment of medaka was conducted under identical conditions of the TECAM by putting 2 medaka into one flask. During the kinetic study, TECAM and medaka were removed after each $1 \mathrm{~h}$ exposure, then put into a new flask filled with $1 \mathrm{~L}$ PAH-spiked water. All the experiments were carried out in triplicates, in the dark to avoid photodegradation of PAHs.

\subsection{Impact of HA on the uptake of acenaphthene and chrysene by medaka and TECAM}

Humic acid stock solution was prepared by dissolving commercial HA purchased from Fluka (Switzerland) in $0.01 \mathrm{~mol} / \mathrm{L}$ sodium hydroxide solution $(\mathrm{pH}=12)$, being sonicated for $30 \mathrm{~min}$ and stirred for about $24 \mathrm{~h}$. Then it was filtered to prerinsed $0.45-\mu \mathrm{m}$ cellulose acetate nitrate membrane filters to remove remaining particulate material and the organic carbon content of the solution was measured with a total organic carbon analyzer (phoenix-8000, Tekmar-Dohrmann, USA). Humic acid concentrations are expressed as milligrams of organic carbon per liter of water. The solution was stored in the dark at $4^{\circ} \mathrm{C}$ until used.

The sample solutions containing HA were produced through diluting the humic acid stock solution with ultrapure water to reach organic carbon content of 0 (i.e. pure water serving as the DOC-free control), 1, 2, 5, 10, $15 \mathrm{mg} \mathrm{C} \cdot \mathrm{L}^{-1}$, respectively, and then they were adjusted to a $\mathrm{pH}$ of $7.5 \pm 0.3$ with $0.1 \mathrm{~mol} \cdot \mathrm{L}^{-1} \mathrm{HCl}$ and 0.1 $\mathrm{mol} \cdot \mathrm{L}^{-1} \mathrm{NaOH}$. One liter of each solution was transferred to each 1-L conical flask and spiked with two test PAHs to give a nominal concentration of $100 \mathrm{ng} \cdot \mathrm{L}^{-1}$. The flasks were kept sealed with Teflon-lined caps and shaken gently on the shaker for $4 \mathrm{~d}$ in the dark to establish equilibrium for PAHs between HA and aqueous phase $^{[2]}$. Following the equilibration, one medaka was introduced into each flask, and the test flasks were sealed and kept static at $25^{\circ} \mathrm{C}$. Exposures were lasted for $12 \mathrm{~h}$. TECAM accumulation study was performed under identical conditions with medaka uptake experiment. One piece of TECAM was immersed in each flask of sample solution. The flasks were sealed and shaken for $12 \mathrm{~h}\left(75 \mathrm{r} \cdot \mathrm{min}^{-1}, 25^{\circ} \mathrm{C}\right)$. During the exposure, the sample solution in flasks was renewed every hour and thus 11 successive renewals were required.

\subsection{Sample processing}

Prior to the extraction, all medaka were rinsed with distilled water and wiped up with hexane dipped pledget to remove the sorptive particles on the surface. After that they were exenterated and weighed. Then the fish were ground with $5 \mathrm{~g}$ of precombusted sodium sulfate each 
and dried. The resulting powder was ultrasonic extracted with $10 \mathrm{~mL}$ of $n$-hexane/dichloromethane $(1: 1, v / v)$ for $10 \mathrm{~min}$. The solvent was replaced and the extraction was repeated twice. The $30 \mathrm{~mL}$ distilled solution was combined and centrifuged for $10 \mathrm{~min}$. The supernatant was reduced by rotary evaporation to approximately $1 \mathrm{~mL}$ and subjected to an alumina/silica gel column for clean-up and fractionation. The column was eluted with $70 \mathrm{~mL}$ of dichloromethane/hexane $(30: 70, v / v)$. The eluate was concentrated to $0.5 \mathrm{~mL}$ under a gentle stream of $\mathrm{N}_{2}$. An appropriate amount of internal standard (hexamethylbenzene) was added and the extract was concentrated under a gentle stream of $\mathrm{N}_{2}$ again to 0.2 $\mathrm{mL}$ just prior to injection into a gas chromatography (GC).

TECAMs were rinsed with distilled water and wiped up with clean paper tissue. Each TECAM was ultrasonic extracted three times with $10 \mathrm{~mL}$ of hexane/dichloromethane $(1: 3, v / v)$ solvent for 10 -min cycles. After extraction, solutions were combined and concentrated to $0.5 \mathrm{~mL}$ under a gentle stream of $\mathrm{N}_{2}$ and solvent-exchanged into $n$-hexane. An appropriate amount of internal standard (hexamethylbenzene) was added to the final concentrated extract prior to instrumental analysis.

\subsection{Instrumental analysis}

PAHs were analyzed using an Agilent 6890 series gas chromatograph, equipped with an Agilent 5973 massselective detector (MSD) and a DB-5 MS silica fused capillary column $(60 \mathrm{~m} \times 0.25 \mathrm{~mm}$ inner diameter $\times 0.25$ $\mu \mathrm{m}$ film thickness). Splitless injection of $1 \mu \mathrm{L}$ of sample was automatically conducted with $99.999 \%$ helium as the carrier gas at a constant flow rate of $1 \mathrm{~mL} \cdot \mathrm{min}^{-1}$. Detection was performed using selected-ion monitoring. The GC oven temperature was programmed from $50^{\circ} \mathrm{C}$ (2 min) to $200^{\circ} \mathrm{C}(2 \mathrm{~min})$ at $20^{\circ} \mathrm{C} \cdot \mathrm{min}^{-1}$, then to $240^{\circ} \mathrm{C}$ $\left(2 \mathrm{~min}\right.$ ) at $5^{\circ} \mathrm{C} \cdot \mathrm{min}^{-1}$ before reaching $310^{\circ} \mathrm{C}$ at $3{ }^{\circ} \mathrm{C}$. $\mathrm{min}^{-1}$ and held for $6 \mathrm{~min}$. The injector and detector temperatures were $310{ }^{\circ} \mathrm{C}$ and $250{ }^{\circ} \mathrm{C}$, respectively. PAHs in the samples were identified by comparing their GC retention times with those of the certified standards, and their identities were further confirmed by matching their GC-MS spectra with those available in the National Institute of Standards and Technology (NIST) MS Library. Quantitation was performed using the internal calibration method based on a six-point calibration curve for individual components with hexamethylbenzene as an internal standard.

\subsection{Quality control}

Method blanks and procedural blanks were routinely analyzed. Surrogate standard phenanthrene- $\mathrm{d}_{10}$ was added to one matrix spike sample for each batch of samples to monitor matrix effects. The recoveries for the two PAHs varied from $63 \%-77 \%$ in fish and $70 \%-85 \%$ in TECAM, respectively. Measured PAH concentrations were corrected for the recovery of PAH matrix spikes. No test PAHs were detected from the blank samples.

\section{Results and discussion}

\subsection{Optimization of negligible depletion extraction}

Agitation is an important parameter in TECAM performance during extraction. For the chemicals studied, agitation significantly increased their uptake rate constants by TECAM. The concentrations of acenaphthene and chrysene in TECAM at maximum agitation (150 $\mathrm{r} \cdot \mathrm{min}^{-1}$ ) were about 10.4 and 7.9 times higher than those at static state after $1 \mathrm{~h}$ exposure, respectively. This is likely due to the fact that mass-transfer resistance of PAHs partitioning from the aqueous phase to TECAM is directly proportional to boundary layer thickness and agitation may decrease the thickness of the unstirred boundary layer adjacent to the TECAM, thereby suggesting an efficient way to accelerate the extraction. Considering the load limit of the shaker and actual operation, 75 $\mathrm{r} \cdot \mathrm{min}^{-1}$ was fixed as the shake speed in subsequent experiments. It was observed that either one piece of TECAM or two medaka exposed for $1 \mathrm{~h}$ accumulated PAHs less than $5 \%$ of the total quantity in the $1 \mathrm{~L}$ exposure solution (Figure 1). The loss of total target chemi-

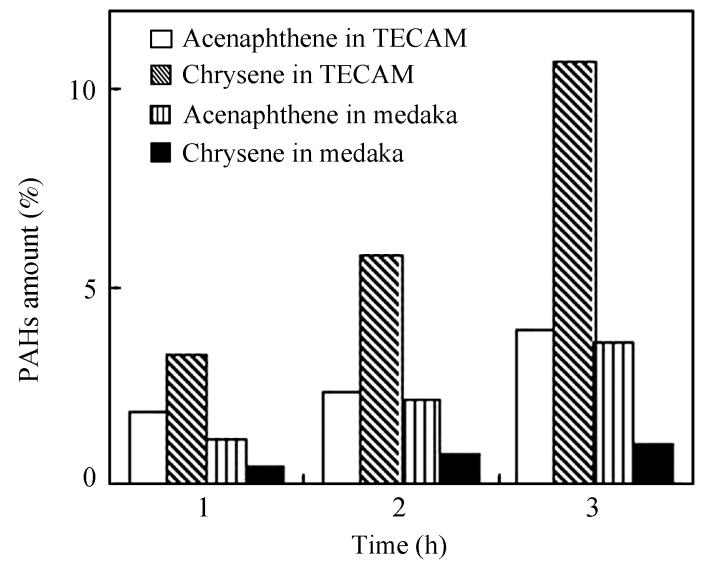

Figure 1 Proportion of two PAHs in TECAM and fish with different exposure time. 
cals in the exposure solution was less than $10 \%$ after $1 \mathrm{~h}$ exposure. Therefore, it could be considered that the concentration of freely dissolved PAHs was relatively constant and the equilibrium existing between the bound and free forms of the chemicals in the solution was not disturbed. Thus the requirement of "negligible depletion" was satisfied. Consequently, all sample solutions during the exposure should be renewed every hour to compensate for the loss of analytes in solution due to accumulation, degradation, volatilization and adsorption to the flask wall.

\subsection{Uptake of PAHs by TECAM and medaka}

During the first accumulation phase in water systems free of DOC, the target chemical elimination from TECAM is negligible and the concentration rises linearly with time. Uptake of PAHs in TECAMs can be simply expressed by the first-order linear model ${ }^{[9]}$.

$$
C_{\mathrm{TECAM}}=C_{\mathrm{w}} k_{\mathrm{u}} t,
$$

where $C_{\text {TECAM }}$ is the concentration of analyte in the TECAM (ng $\left.\cdot \mathrm{g}^{-1}\right), C_{\mathrm{w}}$ is the concentration of analyte in water $\left(\mathrm{ng} \cdot \mathrm{g}^{-1}\right), k_{\mathrm{u}}$ is the uptake rate constant $\left(\mathrm{L} \cdot \mathrm{h}^{-1} \cdot \mathrm{g}^{-1}\right)$, and $t$ is the uptake time (h). As shown in Figure 2, in this experiment the uptake of both PAHs in TECAM reveals good linear correlation to exposure time with $R^{2}$ being

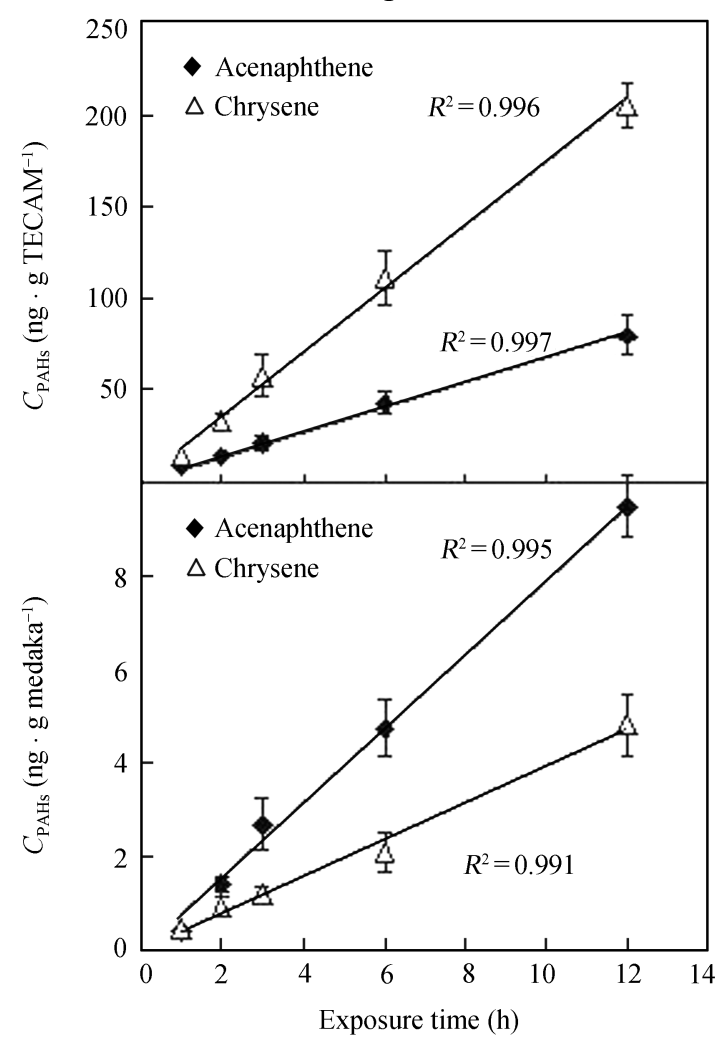

Figure 2 Uptakes of the two PAHs in TECAM and medaka. higher than 0.9. The concentrations of PAHs in medaka were proportional to exposure time, with $R^{2}$ being higher than 0.9 as well. Hence, under laboratory controlled conditions, uptake of PAHs in both sampling matrices remained in the linear phase, and the uptake rate constant $k_{\mathrm{u}}$ was constant during the whole exposure.

\subsection{Impact of HA on the uptake rate constant by medaka and TECAM}

The result of the 12-h bioaccumulation experiment indicated that PAHs accumulation by medaka and TECAM consistently decreased with increasing concentration of HA in sample solutions (Figure 3). For instance, compared to the control sample solution, the average concentration of chrysene in medaka was reduced from 3.43 to $0.38 \mathrm{ng} \cdot \mathrm{g}^{-1}$ in solution containing $15 \mathrm{mg} \mathrm{C} \cdot \mathrm{L}^{-1}$ content of HA, decreasing by $89 \%$, while acenaphthene was reduced from 8.86 to $5.90 \mathrm{ng} \cdot \mathrm{g}^{-1}$, decreasing by $33 \%$. Concurrent analysis of PAHs accumulation in TECAMs showed that $C_{\text {TECAM }}$ decreased with increasing concentration of HA in a trend similar to that of medaka (Figure $3)$. This result suggests that the TECAM sampling method can mimic the uptake of PAHs in medaka

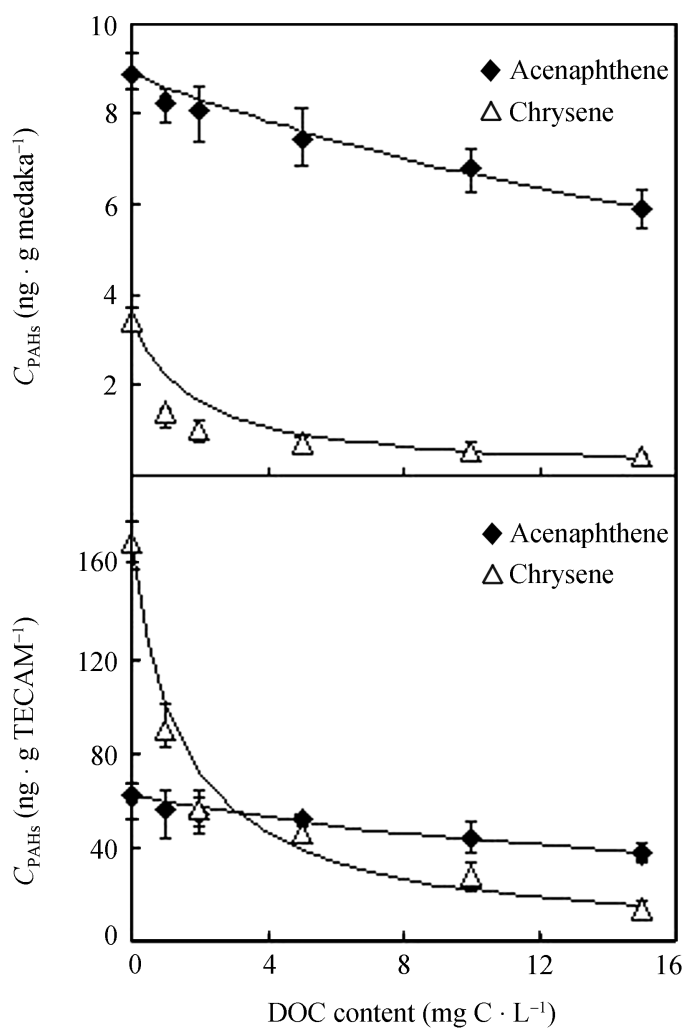

Figure 3 Chrysene and acenaphthene accumulated in Medaka and TECAM exposed in solutions containing HA at different levels; - , Fixed curves. 
in the presence of HA.

Since medaka were not fed before the experiment, PAHs assimilated in peptic during exposure would obviously not affect the amount of PAHs accumulated in medaka. Moreover, as the fish were exenterated prior to weighing, interference of PAHs accumulated via the dietary uptake route was negligible. Therefore, it can be considered that the majority of PAHs accumulated in medaka is regulated by freely dissolved form partitioning from the water phase via gill respiration, i.e. the bioavailable concentration. Hereby, $k_{\mathrm{u}}$ in eq. (1) represents the actual uptake rate constant of freely dissolved compounds by medaka:

$$
k_{\mathrm{u}}=\frac{C_{\text {fish }}}{C_{\text {free }} t},
$$

where $C_{\text {fish }}$ is the concentration of analyte in the medaka $\left(\mathrm{ng} \cdot \mathrm{g}^{-1}\right), C_{\text {free }}$ is the freely dissolved concentration of PAHs in the sample solutions $\left(\mathrm{ng} \cdot \mathrm{g}^{-1}\right)$. If HA is present in the solutions in a dissolved state, and the amount of PAHs accumulated in medaka will be reduced due to a reduced freely dissolved fraction in the water phase. Then the relationship between PAHs concentration in medaka and total concentration in solutions can be given as follows:

$$
C_{\text {fish }}=F C_{\mathrm{w}} k_{\mathrm{u}} t,
$$

with $F$ being the fraction freely dissolved analyte to total amount according to eq. (4) $)^{[15] \text { : }}$

$$
F=\frac{1}{1+K_{\mathrm{DOC}}[\mathrm{DOC}]},
$$

where $K_{\mathrm{DOC}}$ is the partition coefficient for DOC, [DOC] is the dissolved organic carbon concentration in solution. To examine the potential attenuating effects of DOC on PAHs uptake in medaka, $k_{\mathrm{u}}^{\text {obs }}$ is defined as apparent uptake rate constant:

$$
\frac{k_{\mathrm{u}}}{k_{\mathrm{u}}^{\mathrm{obs}}}=\frac{1}{F}=1+K_{\mathrm{DOC}}[\mathrm{DOC}] .
$$

By determining the $k_{\mathrm{u}}^{\mathrm{obs}}$ based on total PAHs concentration contained in the solution with HA, deviation between the true uptake rate constant $k_{\mathrm{u}}$ and $k_{\mathrm{u}}^{\text {obs }}$ versus [DOC] is given by eq. (6):

$$
\frac{k_{\mathrm{u}}}{k_{\mathrm{u}}^{\mathrm{obs}}}=\frac{1}{F}=1+K_{\mathrm{DOC}}[\mathrm{DOC}] .
$$

Similarly, TECAM $k_{\mathrm{u}}^{\text {obs }}$ can be expressed using the above model supposing only freely dissolved PAHs could be accumulated by TECAMs.
Considering the same exposure time frame for fish and TECAMs with constant total concentrations of PAHs in sample solutions, the reduction trend of PAHs concentrations in the two sampling matrices with increasing HA levels shown in Figure 3 also represented the reduction trend of apparent uptake rate constant. Meanwhile, uptake conditions did not change in either TECAM or fish exposure experiments, so the actual uptake rate constant of $k_{\mathrm{u}}$ should be independent of HA concentrations in sample solutions. The relationship between experimental data of $k_{\mathrm{u}} / k_{\mathrm{u}}^{\mathrm{obs}}$ and [DOC] is validated in Figure 4. Here a good fitness was observed for both samplers with $R^{2}$ above 0.9 , illustrating that the presence of HA in exposure solutions reduced medaka and TECAM $k_{\mathrm{u}}^{\mathrm{obs}}$ to different extent for two test PAHs in the linear uptake phase. When referred to individual chemical, the deviation of $k_{\mathrm{u}}^{\text {obs }}$ from true $k_{\mathrm{u}}$ got larger throughout the increased HA concentration series.

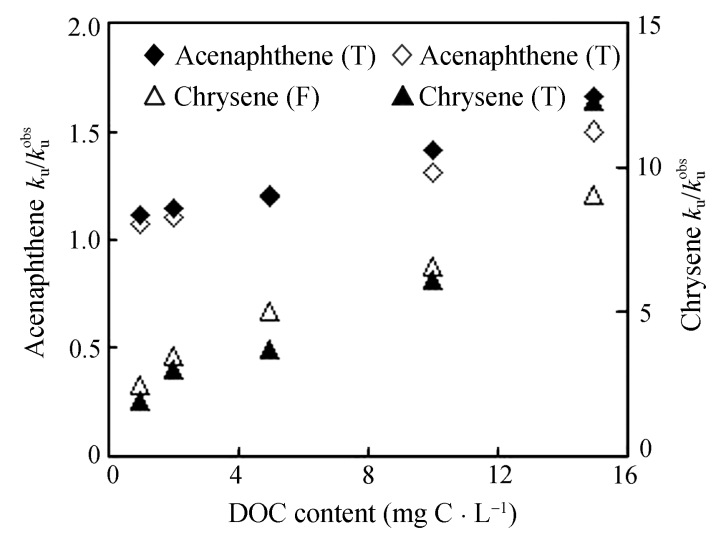

Figure 4 Deviation of $k_{\mathrm{u}}^{\mathrm{obs}}$ from $k_{\mathrm{u}}$ in medaka and TECAM exposed in solutions containing HA at different levels.

Fitting the experimental accumulation data of medaka and TECAM to eq. (6) resulted in the $\log K_{\text {DOC }}$ values listed in Table 1. Those values are in good agreement with the results reported previously in literatures. This fact indicates that TECAM only accumulated the freely dissolved fraction of PAHs in water and, accordingly, could determine adequately the bioavailable fraction of PAHs in the aqueous environment. Several studies have shown that $K_{\mathrm{DOC}} \mathrm{S}$ of a single class of compounds seemed to be proportional to their lipophilicities ${ }^{[4,10,16]}$. Chrysene is more likely to partition into the DOC phase than acenaphthene due to its stronger lipophilicity. Because the effect of DOC on the uptake is a result of both the amount of DOC and $K_{\mathrm{DOC}}$, the same concentration of DOC in solution should lead to greater reduction 
of truly dissolved concentration for chrysene than acenaphthene. As a consequence, the reduction in the uptake of acenaphthene would not be as markedly signifycant as chrysene at the DOC concentrations used in the present study, especially at low DOC levels. The variation trend of chrysene appeared distinctly curvilinear while acenaphthene approximately linear (Figure 3), which was consistent with the theoretical conclusion presented above.

Table 1 Partition coefficients $\left(K_{\mathrm{DOC}}\right)$ of PAHs measured by TECAM and medaka

\begin{tabular}{lccc}
\hline \multicolumn{1}{c}{ PAHs } & $\log K_{\mathrm{DOC}}^{\text {a) }}$ & $\log K_{\mathrm{DOC}}{ }^{\text {b) }}$ & $\log K_{\mathrm{DOC}}{ }^{\mathrm{c})}$ \\
\hline Acenaphthene & 4.2 & 4.63 & 4.52 \\
Chrysene & 5.3 & 5.83 & 5.76 \\
\hline
\end{tabular}

a) Values from ref. [19]; b) values calculated by uptake rate in medaka; c) values calculated by uptake rate in TECAMs.

Chiou et al. ${ }^{[17]}$ have found that the triolein is a major lipid in fishes and accumulation pattern of HOCs in triolein is similar to that in fat reserves of fish. In the light of the result in this study, it was clearly proved that the TECAM sampling method meets all the characters to the definition of biomimetic sampling, including passive pattern, accumulation ${ }^{[18]}$, negligible depletion and representing freely dissolved fraction. Since the bioavailability of HOCs is largely controlled by diffusion and

1 Wershaw R L. Model for humus in soils and sediments. Environ Sci Technol, 1993, 27(5): 814-816

2 Akkanen J, Kukkonen J V K. Measuring the bioavailability of two hydrophobic organic compounds in the presence of dissolved organic matter. Environ Toxicol Chem, 2003, 22(3): 518-524

3 Landrum P F, Reinhold M D, Nihart S R, et al. Predicting the bioavailability of organic xenobiotics to pontoporeia hoyi in the presence of humic and fulvic materials and natural dissolved organic matter. Environ Toxicol Chem, 1985, 4(4): 459-467

4 Mott H V. Association of hydrophobic organic contaminants with soluble organic matter: Evaluation of the database of $K_{\text {doc }}$ values. Adv Environ Res, 2002, 6: 577-593

5 Menzie C A, Potocki B B, Santodonato J. Exposure to carcinogenic PAHs in the environment. Environ Sci Technol, 1992, 26(7): $1278-1284$

6 Li Q L, Xu X Q, Lee F S, et al. Determination of trace PAHs in seawater and sediment pore-water by solid-phase microextraction (SPME) coupled with GC/MS. Sci China Ser B-Chem, 2006, 49(6): $481-491$

7 Wei C, Frank S, Weiping L, et al. Effects of dissolved organic matter on permethrin bioavailability to Daphnia species. Agric Food Chem, 2006, 54(11): 3967-3972

8 Södergren A. Solvent-filled dialysis membranes simulate uptake of pollutants by aquatic organisms. Environ. Sci Technol, 1987, 21(9): $855-863$

9 Ke R H, Xu Y P, Wang Z J, et al. Estimation of the uptake rate constants for polycyclic aromatic hydrocarbons accumulated by semipermeable membrane devices and triolein-embedded cellulose acetate membranes. Environ Sci Technol, 2006, 40(12): 3906-3911

10 Miège $\mathrm{C}$, Ravelet $\mathrm{C}$, Croue $\mathrm{J} \mathrm{P}$, et al. Semi-permeable membrane device efficiency for sampling free soluble fraction of polycyclic aromatic hydrocarbons. Anal Chim Acta, 2005, 536(1-2): 259-266 partitioning processes, it is clear that the TECAM sampling method was similar to biomimetic sampling. Thus TECAM can serve as a standardized "biomimetic" tool to determine the bioavailable concentration and predict the bioavailability of HOCs in the aqueous environment.

\section{Conclusions}

The presence of HA in water decreases $k_{\mathrm{u}}^{\mathrm{obs}}$ in medaka for acenaphthene and chrysene, thus resulting in reduction of concentrations accumulated in organisms. The ratio of the true uptake rate constant $k_{\mathrm{u}}$ to $k_{\mathrm{u}}^{\text {obs }}$ highly correlated with HA contents in exposure solutions $\left(R^{2}>\right.$ $0.9)$, suggesting that HA sorption will reduce freely dissolved portion of PAHs in water phase and result in reduced bioavailability.

As verified in the present study, only the freely dissolved PAHs can be accumulated by the TECAM. Thus, it can be used as a surrogate to evaluate the impact of waterborne HA on the uptake of PAHs in medaka. With the help of the calculated $K_{\mathrm{DOC}}$ value by regression fit, it is possible to accurately measure freely dissolved compounds. Therefore, TECAM shows great promise to work as a biomimetic sampler to quantitatively predict impact of DOC on bioavailability of PAHs in the aqueous environment to fishes.

11 Huckins J N, Tubergen M W, Manuweera G K. Semipermeable membrane devices containing model lipid: A new approach to monitoring the bioavailability of lipophilic contaminants and estimating their bioconcentration factor. Chemosphere, 1990, 20(5): $533-552$

$12 \mathrm{Xu}$ Y P, Wang Z J, Ke R H, et al. Accumulation of organochlorine pesticides from water using triolein embedded cellulose acetate membranes. Environ Sci Technol, 2005, 39(4): 1152-1157

13 Poerschmann J, Zhang Z Y, Kopinke F D, et al. Solid phase microextraction for determining the distribution of chemicals in aqueous matrices. Anal Chem, 1997, 69(4): 597-600

$14 \mathrm{Xu} \mathrm{Y} \mathrm{P,} \mathrm{Lv} \mathrm{Y} \mathrm{B,} \mathrm{Li} \mathrm{J,} \mathrm{et} \mathrm{al.} \mathrm{The} \mathrm{research} \mathrm{on} \mathrm{the} \mathrm{preparation} \mathrm{and}$ fundamental properties of a new type of cellulose acetate composite membrane. High TechnoL Lett (in Chinese), 2004, 14(1): 89-94

15 Anderson P F, Elsa A G, Frans J B, et al. Estimating impact of humic acid on bioavailability of hydrophobic chemicals in guppies using kinetic solid-phase extraction. Environ Toxicol Chem, 1998, 17(6): $998-1004$

16 Huo J X, Liu H J, Qu J H, et al. Dieldrin and endrin removal from water by triolein-embedded adsorbent. Chin Sci Bull, 2005, 50(23): 2696-2700

17 Chiou C T. Partition coefficients of organic compounds in lipid-water systems and correlations with fish bioconcentration factors. Environ Sci Technol, 1985, 19(1): 57-62

18 Ke R H, Qiao M, Xu Y P, et al. Comparison between biomimetic sampling technique using semipermeable membrane device and bioconcentration in caged fish for polycyclic aromatic hydrocarbons. Chinese J Environ Sci (in Chinese), 2006, 27(7): 1410-1414

19 Jeffrey N B, Barrie M P. Determination of colloidally-associated polycyclic aromatic hydrocarbons (PAHs) in fresh water using $\mathrm{C} 18$ solid phase extraction disks. Anal Chim Acta, 2003, 486(2): 159169 